\title{
Imaging Findings of a Primary Cardiac Osteosarcoma Arising From the Left Atrium and Mitral Posterior Leaflet with Multiple Metastases in A 58-Year-Old Woman
}

\author{
Eun Jee Song, ${ }^{1}$ Se Hwan Kwon,, ${ }^{,}$Hyo Chul Youn, ${ }^{3}$ Soo Cheol Kim, ${ }^{3}$ Jong Soo Shin, ${ }^{4}$ and Joo Hyeong $\mathrm{Oh}^{2}$ \\ ${ }^{1}$ Department of Medicine, Graduate School, Kyung Hee University, Seoul, Republic of Korea \\ ${ }^{2}$ Department of Radiology, College of Medicine, Kyung Hee University Hospital, Kyung Hee University, Seoul, Republic of Korea \\ ${ }^{3}$ Department of Cardiothoracic Surgery, College of Medicine, Kyung Hee University, Seoul, Republic of Korea \\ ${ }^{4}$ Department of Radiology, Kyung Hee University Hospital at Gangdong, Seoul, Republic of Korea \\ "Corresponding author: Se Hwan Kwon, Department of Radiology, College of Medicine, Kyung Hee University Hospital, Kyung Hee University, Seoul, Republic of Korea. Tel: \\ +82-29588622, Fax: +82-29680787, E-mail: Kwon98@khu.ac.kr
}

Received 2015 July 23; Revised 2015 September 07; Accepted 2015 September 15.

\begin{abstract}
Primary cardiac osteosarcoma is a very rare malignancy with nonspecific symptoms, making early diagnosis a challenge. We report the computed tomography and magnetic resonance imaging findings of a primary cardiac osteosarcoma arising from the left atrium and posterior mitral leaflet in a 58-year-old woman with multiple metastases to the brain, skull, clavicle, lung, ribs, liver, lymph nodes, and thyroid gland.
\end{abstract}

Keywords: Cardiac Tumor, Cardiac Osteosarcoma, Metastasis, Surgery, Computed Tomography, Magnetic Resonance Imaging

\section{Introduction}

Primary cardiac tumors are found in $0.002 \%-0.3 \%$ of patients in autopsy studies (1). A primary cardiac osteosarcoma is a clinical and pathological surprise, accounting for less than $10 \%$ of primary cardiac malignancies and only $3 \%$ - $9 \%$ of all cardiac sarcomas $(1,2)$. Unlike metastatic osteosarcoma, which most often occurs in the right atrium, primary cardiac osteosarcomas arise in the left atrium (LA) in the overwhelming majority of cases (2). We describe the computed tomography (CT) and magnetic resonance imaging (MRI) findings of a primary cardiac osteosarcoma arising from the LA and posterior mitral leaflet in a 58-yearold woman with multiple metastases to the brain, skull, clavicle, lung, ribs, liver, lymph nodes, and thyroid gland.

\section{Case Presentation}

A 58-year-old woman was admitted to our hospital complaining of chest discomfort, dyspnea, hoarseness, and a mild headache. Her symptoms had begun one month earlier. The patient denied a history of systemic disease, and her family history, including for malignancy, was non-contributory. An electrocardiogram (ECG) showed a sinus rhythm of $70 \mathrm{bpm}$, and all laboratory findings were within normal limits. A chest x-ray revealed clear lungs with a normal-sized heart. A transthoracic echocardiogram showed a $1.5 \times 1.8 \mathrm{~cm}$ echogenic mass in the lateral wall of the LA adjacent to the posterior leaflet of the mitral valve (not shown).

For further evaluation, cardiac CT was performed using an ECG-gated 128-slice multi-detector CT scanner (Ingenuity; Philips Medical Systems, Best, the Netherlands). Unenhanced CT of the heart showed dense calcification in the tumor, and the contrast-enhanced mid-diastolic phase images showed a broad-based low-attenuation mass in the lateral wall of the LA adjacent to the mitral valve (Figure 1). CT also revealed diffuse thickening of the LA wall around the mass and the posterior leaflet of the mitral valve. No pleural effusion or lymph node enlargement was noted. MRI (Intera Achieva 3T; Philips Medical Systems, Best, the Netherlands) showed a lobulated mass with a broad stalk at the lateral wall of the LA, and diffuse thickening of the adjacent LA wall and posterior leaflet of the mitral valve. The mass was isointense relative to cardiac muscle on T1-weighted images (T1WI), and was hyperintense on T2-weighted images (T2WI) (Figure 2). The mass showed strong enhancement on the contrast-enhanced images.

An initial diagnosis of atrial myxoma was considered. However, the wall thickening that included the posterior mitral leaflet, along with the calcifications, made us consider other malignant cardiac tumors, such as metastasis or sarcoma. After a general evaluation, we decided to respect the tumor. Intraoperatively, the tumor was seen to have invaded the lateral wall of the LA and the posterior mitral leaflet (Figure 3). The atrial mass was resected, but com- 

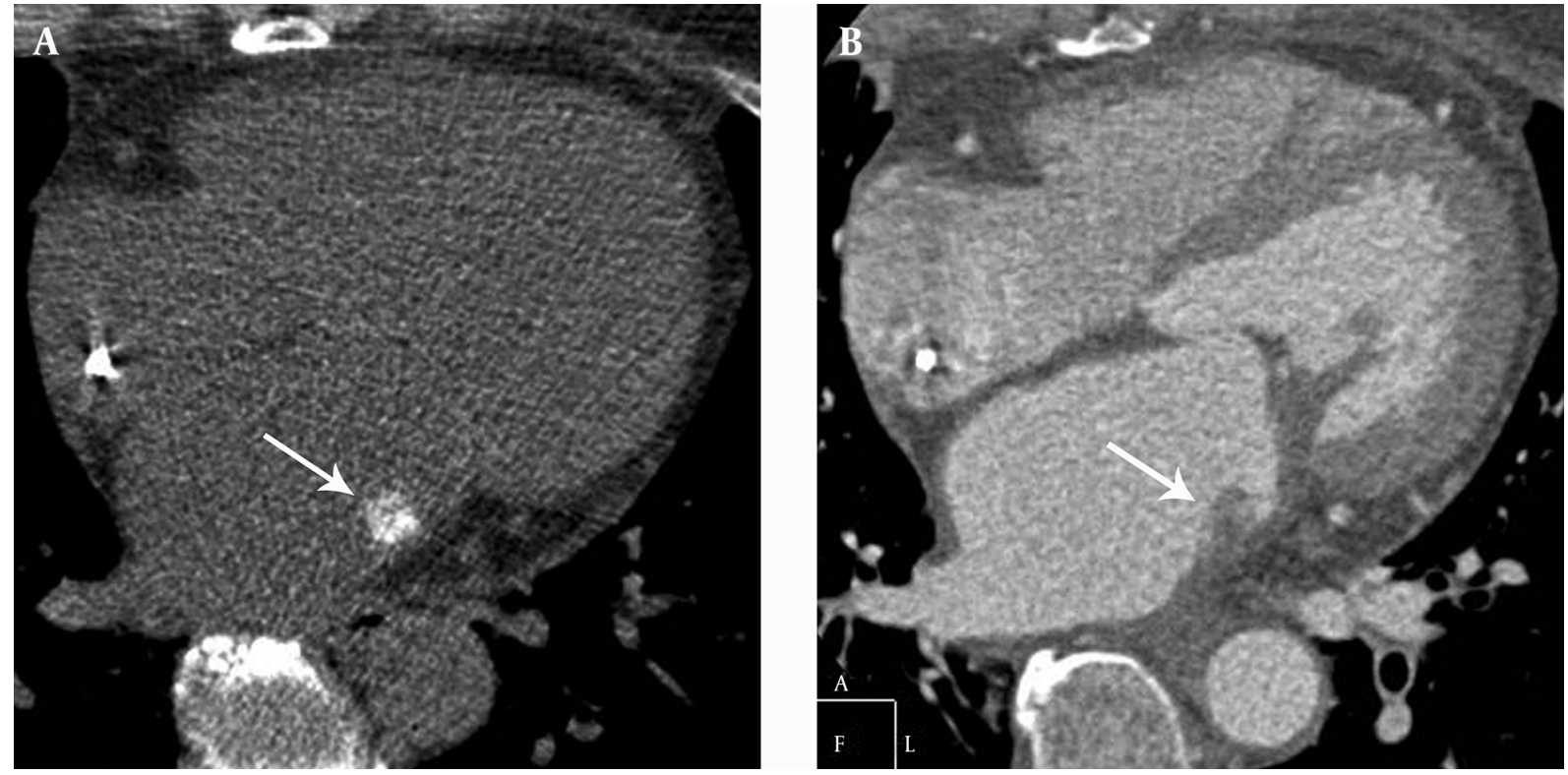

Figure 1. Unenhanced (A) and contrast-enhanced (B) mid-diastolic-phase CT images showing a broad-based tumor (arrows) with dense calcification involving the LA lateral wall, with diffuse thickening of the surrounding LA wall and posterior leaflet of the mitral valve.

plete resection and mitral valve replacement could not be performed due to tumor extension into the adjacent structures. Histologically, the LA mass was composed mainly of atypical spindle-shaped cells forming lace-like osteoid (Figure 4). The final diagnosis was primary cardiac osteosarcoma.

At that time, expansile osteolytic masses in the parietal bone were found on brain CT, measuring $2.0 \times 2.0$ $\mathrm{cm}$ (right) and $4.0 \times 3.3 \mathrm{~cm}$ (left). A craniectomy and mass removal was performed, which confirmed metastatic osteosarcoma. The patient began chemotherapy with ifosfamide and doxorubicin. However, one year later, she presented with multiple metastases, including in the brain, skull, clavicle, lung, ribs, liver, lymph nodes, and thyroid gland. Positron emission tomography (PET)-CT showed multiple fluorodeoxyglucose (FDG)-avid areas in the metastatic lesions (Figure 5). At that time, the patient was admitted to the hospital for supportive care.

\section{Discussion}

Primary cardiac malignancies are rare, and most cardiac tumors are metastases, which are 20 - 40 fold more common than primary tumors $(1,2)$. Benign tumors, such as myxomas, lipomas, and papillary fibroelastomas, comprise approximately $75 \%$ of all primary cardiac tumors, while primary malignant tumors comprise approximately
25\%. Primary cardiac osteosarcoma is rare, accounting for less than $10 \%$ of primary cardiac malignancies (1).

Primary cardiac osteosarcomas are commonly situated in the LA and are frequently confused with LA myxomas on imaging. Possible clinical presentations are similar to those of other mass lesions in the cardiac chambers and may be obstructive, embolic, or metastatic (3). The clinical manifestations of cardiac tumors depend on their anatomical location and reflect the degree of obstruction, invasion, and embolization of specific intracardiac structures. Most patients present with dyspnea secondary to mitral valve obstruction $(3,4)$. Our patient presented with chest discomfort, dyspnea, hoarseness, and a mild headache. In our case, there was debate over whether the cardiac lesion was a primary malignancy, metastatic tumor, or myxoma. Most reported cases of metastatic osteosarcoma are located in the right atrium, and primary osteosarcomas are found in the LA in the overwhelming majority of cases (2). Therefore, the cardiac lesion in our case was thought to be a primary cardiac tumor rather than metastasis. In addition, imaging findings such as a broad base of attachment and an infiltration pattern suggested primary cardiac malignancy rather than benign myxoma (5).

Primary cardiac osteosarcomas are very aggressive, with a high rate of recurrence and metastasis. Metastases are commonly observed at various sites at the time of the initial diagnosis, and the average survival is 6 months from the time of diagnosis $(1,3,6)$. Metastases to distant organs, 

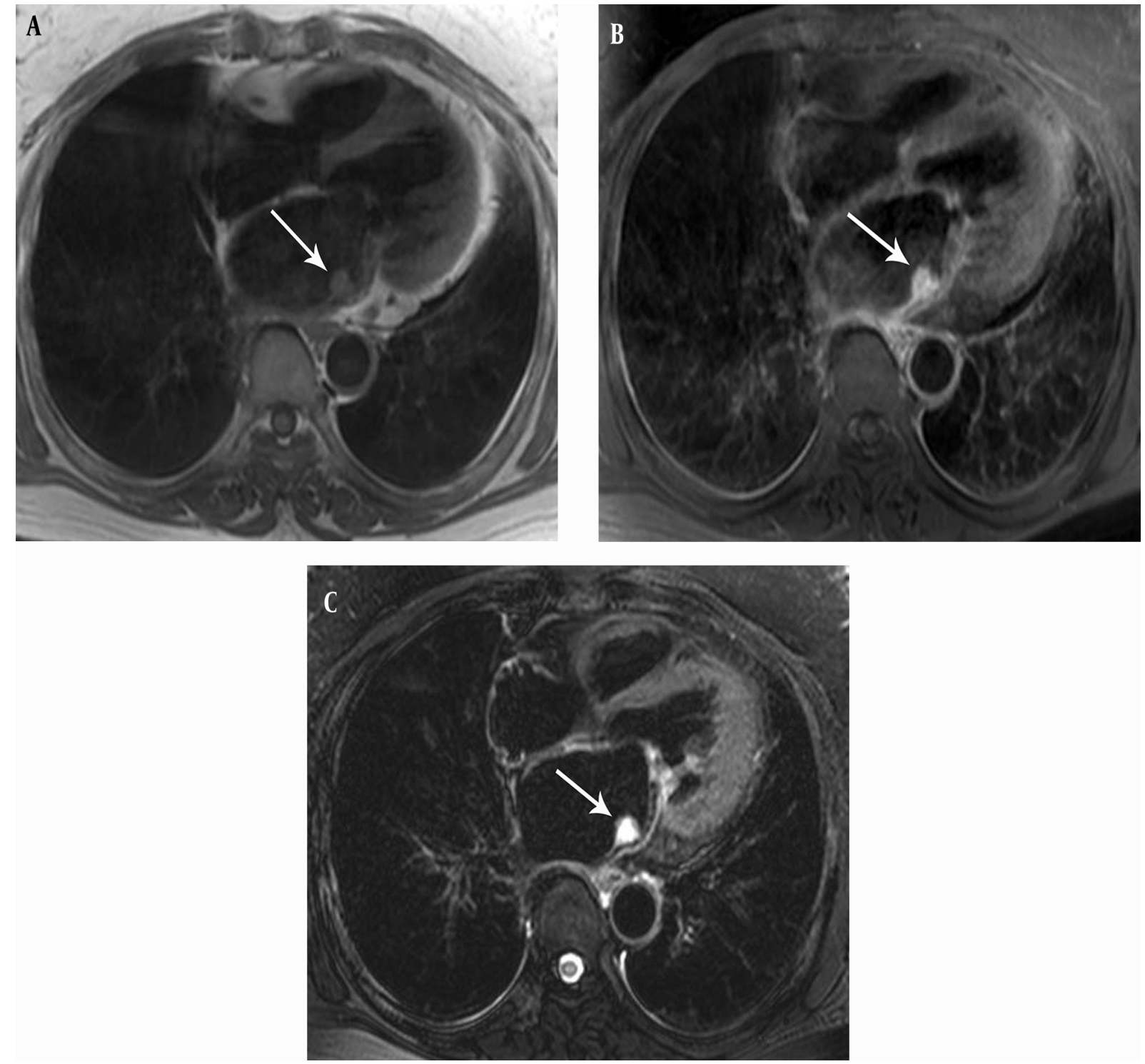

Figure 2. MRI showing a lobulated, broad-based mass (arrows) at the lateral wall of the LA, and diffuse thickening of the surrounding LA wall and posterior leaflet of mitral valve. The mass was isointense relative to cardiac muscle on T1WI (A) and hyperintense on T2WI (B). On the contrast-enhanced images (C), the mass showed strong enhancement.

including the skin, brain, thyroid, lung, stomach, liver, kidney, muscle, intestine, peritoneum, and bone have been reported (4). Our case also revealed multiple metastases in the brain, skull, clavicle, lung, ribs, liver, lymph nodes, and thyroid gland, even though the patient had undergone chemotherapy after surgical removal of the tumor.

According to previous reports, the characteristic CT findings of cardiac osteosarcoma are a low-attenuation mass with dense calcification and a broad base of attachment (6). An aggressive growth pattern may be seen, such as extension or invasion into the surrounding structures (mitral valve, pulmonary veins, atrial septum, and epicardium) (1, 2). However, calcification may also be minimal, and in the early stages it may be mistaken as benign, including degenerative calcification of the mitral valve, caseous calcification of the mitral valve annulus, or another benign, dystrophic type of calcification (2). Our case showed relatively typical CT findings of osteosarcoma, including a densely calcified low-attenuation mass with diffuse thickening of the surrounding LA wall and posterior 


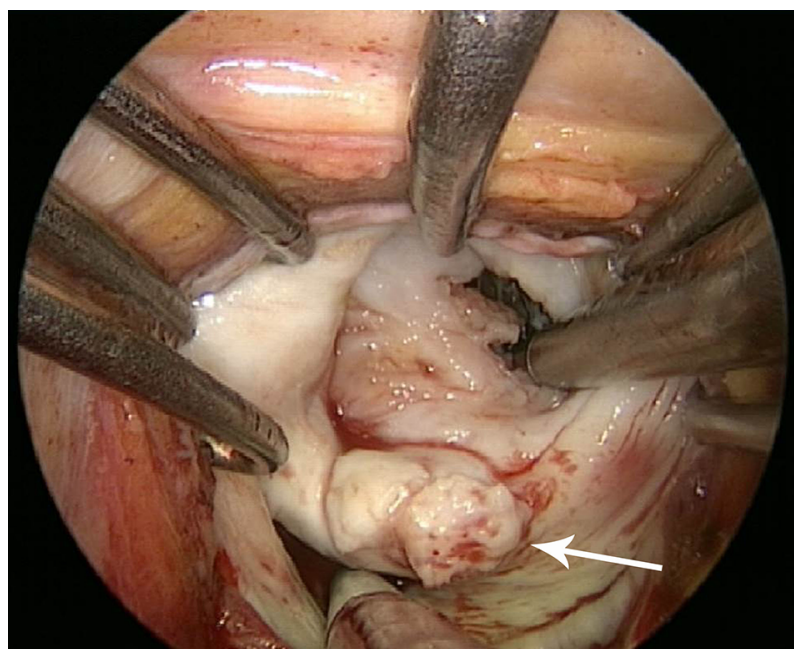

Figure 3. Intraoperative image showing the broad-based LA mass (arrow) with diffuse thickening of the surrounding LA wall and posterior leaflet of the mitral valve.

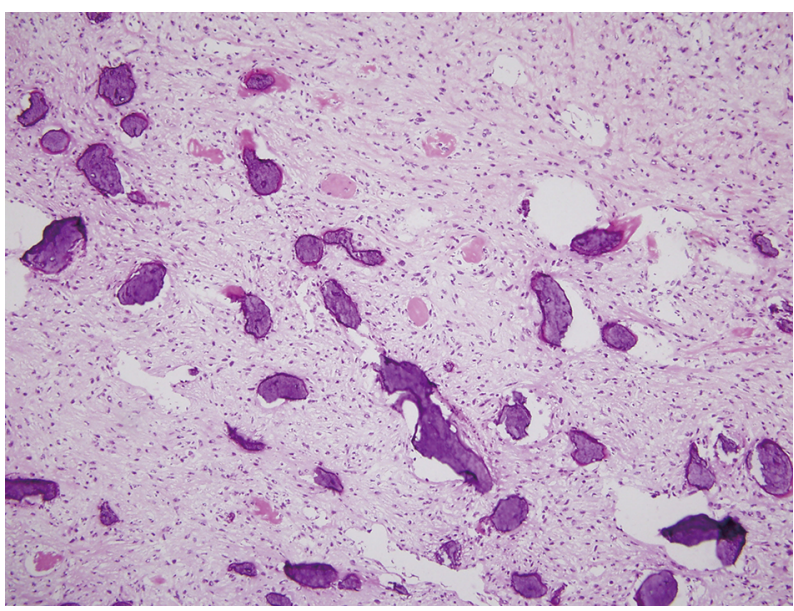

Figure 4. Microscopic image showing atypical round and spindle-shaped cells forming lace-like osteoid

mitral leaflet.

The MRI characteristics of cardiac osteosarcomas still remain unclear. Yamagishi et al. (5) first reported the MRI findings of a cardiac osteosarcoma in which the tumor appeared as a huge mass of heterogeneous signal intensity in the LA. However, their report focused on the role of MRI in differentiating malignant from benign tumors. Cardiac osteosarcomas have been reported as irregularly lobulated masses with variable heterogeneous signal intensity on T1WI and heterogeneous hyperintensity on T2WI $(1,7)$. Ahn et al. (1) reported that cardiac osteosarcomas showed mildly heterogeneous high signal intensity on T1WI and

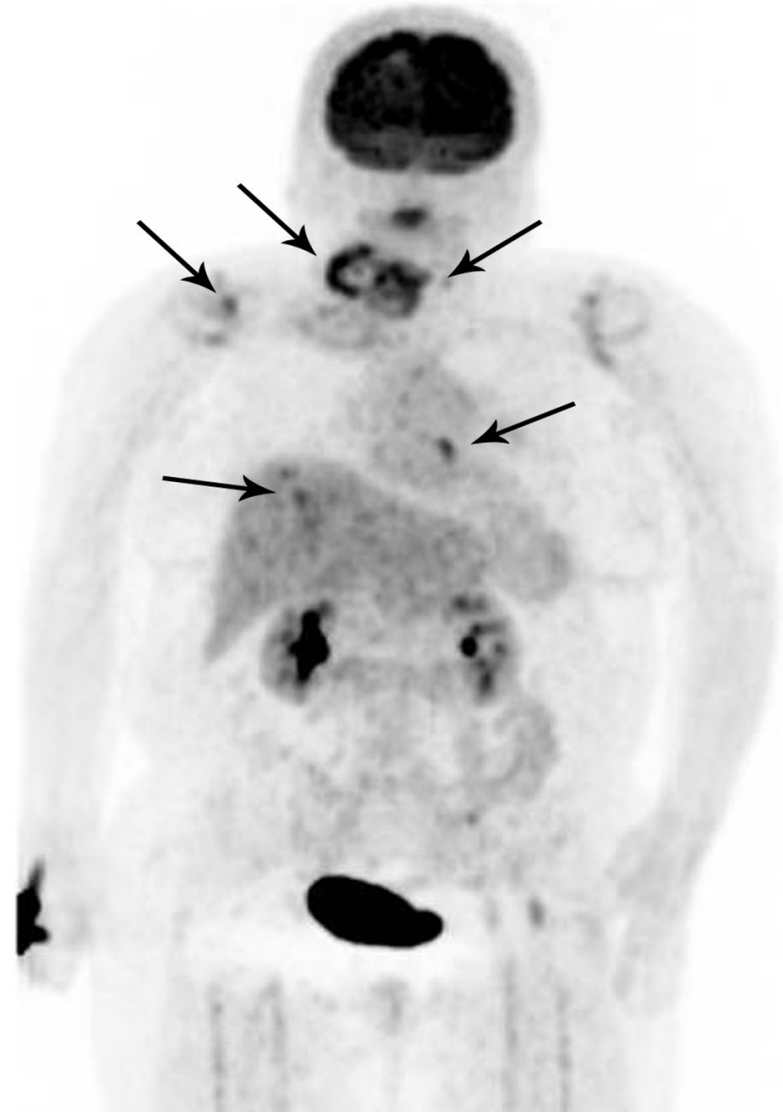

Figure 5. PET-CT image taken one year after surgery, showing multiple FDG-avid areas in the metastatic lesions (arrows).

heterogeneously high signal intensity on T2WI, with heterogeneous enhancement. Foci of hemorrhage may be seen as focal areas of high signal intensity on T1WI, whereas cystic or necrotic changes cause high signal intensity on T2WI. However, hemorrhage and necrosis may be seen on MRI in other sarcomas, making these findings nonspecific (7). In our case, the tumor showed isointense signal relative to cardiac muscle on T1WI, and heterogeneously hyperintense signal on T2WI, with relatively homogeneous enhancement on contrast-enhanced images. Further studies are needed to correlate the signal intensities on MRI images and the contrast-enhancement patterns on CT with the histological composition.

Due to its low incidence, no standard treatment for primary cardiac osteosarcoma has yet been established. Surgical resection is usually the initial treatment. Complete surgical excision is important for improving the survival rate and prognosis of patients with primary cardiac sarcoma $(8,9)$. Unfortunately, in our case, the tumor was not 
resected completely because it had extended into adjacent structures, including the LA wall and the posterior mitral valve leaflet. As a result, our patient developed multiple metastases despite undergoing chemotherapy.

In summary, we present the CT and MRI findings of a rare case of primary cardiac osteosarcoma arising from the LA and posterior mitral leaflet in a 58-year-old woman with multiple metastases to the brain, skull, clavicle, lung, ribs, liver, lymph nodes, and thyroid gland.

\section{Footnotes}

Authors' Contribution: Study concept and design: E] Song, SH Kwon, JH Oh; acquisition of data: EJ Song, HC Youn, SC Kim, JS Shin; analysis and interpretation of data: EJ Song, SH Kwon, HC Youn, SC Kim, JS Shin, JH Oh; drafting of the manuscript: EJ Song, HC Youn, SC Kim; critical revision of the manuscript for important intellectual content: SH Kwon; statistical analysis: none; administrative, technical, and material support: HC Youn, SC Kim, JS Shin; study supervision: SH Kwon, JH Oh.

Financial Disclosure: None declared. Funding/Support: None declared.

\section{References}

1. Ahn S, Choi JA, Chung JH, Choi H, Chun EJ, Choi SI, et al. MR imaging findings of a primary cardiac osteosarcoma and its bone metastasis with histopathologic correlation. Korean J Radiol. 2011;12(1):135-9. doi: 10.3348/kjr.2011.12.1.135. [PubMed: 21228950].

2. Araoz PA, Eklund HE, Welch TJ, Breen JF. CT and MR imaging of primary cardiac malignancies. Radiographics. 1999;19(6):1421-34. doi:10.1148/radiographics.19.6.g99no031421. [PubMed: 10555666].

3. Dan S, Hodge AJ. Osteogenic sarcoma of the left atrium. Ann Thorac Surg. 1997;63(6):1766-8. [PubMed: 9205182].

4. Aguilar CA, Donet JA, Galarreta CI, Yabar A. A primary cardiac osteosarcoma: Case report and review of the literature. J Cardiol Case. 2013;7(2):29-33.

5. Yamagishi $M$, Yamada $N$, Kuribayashi S. Images in cardiology: Magnetic resonance imaging of cardiac osteosarcoma. Heart. 2001;85(3):311. [PubMed: 11179273].

6. Hashimoto W, Hashizume K, Ariyoshi T, Taniguchi S, Miura T, Kinoshita N, et al. Primary cardiac osteosarcoma with imaging that revealed no calcification. Gen Thorac Cardiovasc Surg. 2011;59(3):184-6. doi:10.1007/s11748-010-0656-1. [PubMed: 21448796].

7. O’Donnell DH, Abbara S, Chaithiraphan V, Yared K, Killeen RP, Cury RC, et al. Cardiac tumors: optimal cardiac MR sequences and spectrum of imaging appearances. AJR Am J Roentgenol. 2009;193(2):377-87. doi: 10.2214/AJR.08.1895. [PubMed: 19620434].

8. Lopez M, Pinto A, Moreno V, Diaz M, Gonzalez Baron M. Primary cardiac osteosarcoma. Clin Transl Oncol. 2008;10(8):515-6. [PubMed:18667384].

9. Karagoz Ozen DS, Ozturk MA, Selcukbiricik F, Esatoglu SN, Turna ZH, Beyaz P, et al. Primary osteosarcoma of the heart: experience of an unusual case. Case Rep Oncol. 2013;6(1):224-8. doi: 10.1159/000351123. [PubMed: 23687493]. 